\title{
Eidgenössische Volksinitiative «Für Transparenz in der Krankenversicherung»
}

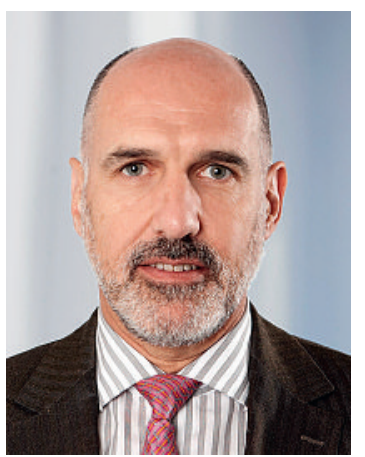

Falls Sie es noch nicht wussten, erfahren Sie es auf Seite 437 dieser Ausgabe: Unterstützt von der FMH lancierten Ärztinnen und Ärzte aus drei Sprachregionen der Schweiz im letzten Herbst eine eidgenössische Volksinitiative, die eine vollständige Trennung zwischen der obligatorischen Krankenversicherung und den privaten Zusatzversicherungen anstrebt. Die Kassen könnten somit künftig nicht mehr beide Versicherungsarten anbieten, für die unterschiedliche gesetzliche Bestimmungen gelten und die mit erheblichen Interessenkonflikten verbunden sind. Die Krankenkassen müssten sich folglich für einen der beiden Versicherungstypen entscheiden.

Von Gesetzes wegen dürfen Krankenkassen richtigerweise keine gewinnorientierten Unternehmen sein, da sie vom Staat mit der Durchführung der sozialen Krankenversicherung beauftragt sind. Der Gesetzgeber wollte damit Marktverzerrungen vermeiden, die sich zwangsläufig ergeben, wenn im Gesundheitsbereich die Gesetze des Marktes und des wirtschaftlichen Wettbewerbs unkontrolliert oder exzessiv zur Anwendung gelangen.

Das derzeitige Gesetz erlaubt den Krankenkassen beide Versicherungsarten - und führt sie damit zu sehr in Versuchung! Die soziale Krankenversicherung und die privaten Zusatzversicherungen sollen nicht mehr unter einem Dach erlaubt sein. Denn egal, ob private Versicherer im Hintergrund sind oder ob die Krankenkassen selbst nach einer Erweiterung ihres Angebots auf die Zusatzversicherungen drängen, entsteht häufig eine Situation, in der das System pervertiert wird.

\section{Die soziale Krankenversicherung und die privaten Zusatzversicherungen sollen nicht mehr unter einem Dach erlaubt sein.}

Je nachdem, ob Sie jung oder alt, gesund oder krank sind, können Sie aufgrund der Risikoselektion die Krankenkasse mit drei Mausklicks problemlos wechseln. Oder Ihr Anruf verbleibt in der Warteschlaufe, Ihr Schreiben geht verloren und die Antwort kommt niemals an. Dasselbe gilt für die Schnelligkeit von Kostenerstattungen, für das «Tierspayant-Prinzip» in den Apotheken usw. Zudem ist jedem selbst überlassen, gemäss seiner Erfahrung einzuschätzen, ob undichte Stellen zwischen verschiedenen Computern in Bezug auf vertrauliche Daten bestehen ...
Diese Jagd nach guten Risiken, die Selektion von Versicherten und die teilweise schwer nachvollziehbaren Finanzströme (z. B. für die politischen Aktivitäten von santésuisse) sind nicht akzeptabel und müssen untersagt werden. Dies ist das Ziel der eidgenössischen Volksinitiative «Für Transparenz in der Krankenversicherung», von der in dieser Ausgabe der Schweizerischen Ärztezeitung die Rede ist. Die Delegiertenversammlung der FMH hat beschlossen, die Initiative zu unterstützen, und lädt Sie ein, dies ebenfalls zu tun.

\section{Wir rufen Sie auf, die Volksinitiative «Für Transparenz in der Krankenver- sicherung» zu unterstützen und auch von weiteren Personen unterzeichnen}

\section{zu lassen.}

Zu klären bleibt die Frage der Gleichzeitigkeit mehrerer Initiativen.

Die Linken, die Patientenorganisationen und zahlreiche Berufsverbände des Gesundheitsbereichs haben vor kurzem die Volksinitiative «Für eine öffentliche Krankenkasse» lanciert. Die FMH hat zu dieser Initiative noch nicht Stellung genommen. Gemäss den Westschweizer Urhebern der Transparenz-Initiative, um die es hier geht, konkurrenzieren sich die beiden Initiativen nicht. Sie sprechen sich daher für eine Unterstützung beider Vorlagen aus.

$\mathrm{Zu}$ erwähnen ist auch das Projekt der Ostschweizer Kantone für kantonale oder regionale Kassen. Dieses steht noch nicht definitiv fest, ist aber schon sehr präsent in der öffentlichen Diskussion.

Die Debatte im Parlament über diese Initiativen wird sicherlich in Richtung einer Kombination der vorhandenen Optionen und Varianten führen. Die verschiedenen Möglichkeiten für die Entwicklung unseres Krankenversicherungssystems konkurrenzieren sich somit nicht, sondern sie können sich vielmehr ergänzen.

Deshalb rufen wir Sie alle dazu auf, die Volksinitiative «Für Transparenz in der Krankenversicherung» zu unterstützen und von möglichst vielen Personen in Ihrem Umfeld unterzeichnen zu lassen. Im Namen des Initiativkomitees ${ }^{1}$ danken wir Ihnen herzlich für Ihre Unterstützung.

Dr. med. Jacques de Haller, Präsident der FMH

Bertrand Buchs, Franco Denti, Charles Abram Favrod-Coune, Marc-Henri Gauchat, Marcus Lütolf, Jürg Nadig, Remo Osterwalder, Jean-Pierre Pavillon, Pierre-Alain Schneider, Urs Stoffel, Igor Svarin, Pierre Vallon, Paul Olivier Vallotton, Hans-Anton Vogel, Peter Wiedersheim 